\title{
Experiencias de trabajadores del retail en Chile. Aproximación desde la sociología de Dubet
}

\author{
Experiences of Retail-store Workers in Chile. \\ An Approach from Dubet's Sociology
}

\author{
Rodolfo Martinic (D) https://orcid.org/0000-0001-7179-319X \\ EHESS, Francia, rodolfomartinic@gmail.com
}

Antonio Stecher (D) https://orcid.org/0000-0001-6235-1912,

Universidad Diego Portales,Chile, antonio.stecher@udp.cl

Recepción:

22/01/19

Aprobación: $18 / 07 / 19$

\begin{abstract}
The article presents the main findings of research on the social experience of the workers of the Department Stores in the retail sector in Chile, an economic field that has experienced a profound process of modernization and productive restructuring in Latin America since 1990. The research was driven by the theoretical approach of François Dubet's sociology of experience and resorted to a qualitative methodology that included 31 interviews with sales workers of the three major department stores in the country and six interviews with key actors in retail, which were analyzed through the codification procedures of the Grounded theory. The results describe the action logics (integration, strategy and subjectivization) and the specific modalities in which they articulate to give rise to three figures of work experience: engaged, blocked and in transition. The conclusions elaborate on the study's contribution to the field of Labor Studies in Latin America.
\end{abstract}

Key words: sociology of work, social experience, retail, Chile, Dubet.

Resumen: Se presentan los resultados de una investigación sobre la experiencia social que construyen los trabajadores de las tiendas por departamentos del sector del retail en Chile; un sector económico que ha experimentado en América Latina un profundo proceso de modernización y reestructuración productiva desde 1990. Se empleó la perspectiva teórica de la Sociología de la Experiencia de François Dubet. Se utilizó una metodología de naturaleza cualitativa que consideró 31 entrevistas a trabajadores de las tiendas, y seis entrevistas a informantes claves, analizadas mediante los procedimientos de codificación de la teoría fundamentada. Los resultados describen las lógicas de acción (integración, estrategia y subjetivación) que despliegan los trabajadores en las tiendas y las modalidades específicas en que se articulan para dar lugar a tres figuras de la experiencia laboral: comprometida, bloqueada y en tránsito. Se concluye discutiendo el aporte empírico y conceptual del estudio al campo de los estudios laborales en Latinoamérica.

Palabras clave: sociología del trabajo, experiencia social, retail, Chile, Dubet. 


\section{Introducción ${ }^{1}$}

Al igual que en distintos países de América Latina, el mundo del trabajo en Chile ha experimentado significativas transformaciones en las últimas cuatro décadas (De la Garza, 2000; Ramos, 2009). En el marco del proceso mundial de reestructuración capitalista (crisis del modelo fordista-keynesiano y consolidación de un nuevo modelo flexible-neoliberal), se observan profundos cambios a nivel de la organización de los procesos productivos, las estrategias de gestión empresarial, las relaciones laborales, el patrón tecnológico y las culturas laborales (Boltansky y Chiapello, 1998; Castells, 2001; Ramos, 2009).

Como ha sido analizado por los Nuevos Estudios Laborales en América Latina, dichos procesos de reestructuración productiva, llevados a cabo desde los años ochenta en la región y orientados por lógicas flexibilizadoras, están lejos de ser homogéneos, presentan profundas variaciones según los contextos nacionales y los sectores económicos, y coexisten y se articulan en el heterogéneo y desigual paisaje laboral de la región, con lógicas productivas de carácter familiar, artesanal o (neo)taylorista (Antunes, 2003; De la Garza, 2000).

Dicha transformación de los escenarios laborales debe ser comprendida, a su vez, considerando el particular trayecto de modernización de la región, marcado por una posición semiperiférica en la economía mundial y por el tránsito desde el modelo nacional-desarrollista de industrialización por sustitución de importaciones (fordismo periférico), hacia una nueva matriz de desarrollo (neo)exportadora y de impronta neoliberal basada en fuertes procesos de privatización, mercantilización, flexibilización, individualización y apertura comercial a las redes globales (Domingues, 2009).

Dentro de dicho contexto de transformaciones estructurales del mundo del trabajo, una línea relevante de la investigación social se ha orientado a comprender el impacto de dichos cambios económicos y sociolaborales a nivel de las experiencias e identidades de los trabajadores (Battistini, 2004;

1 Este artículo tiene el apoyo y fue posible gracias al financiamiento del Fondo Nacional de Desarrollo Científico y Tecnológico del gobierno de Chile (FONDECYT-CONICYT) a través de los proyectos Fondecyt $\mathrm{N}^{\circ} 11130095$, "Procesos de construcción de identidad en el trabajo en el Chile actual: El caso de los trabajadores de tienda de grandes empresas del Retail. Aportes empíricos y conceptuales al debate sobre trabajo e identidad en América Latina” y Fondecyt $\mathrm{N}^{\circ} 1181041$ “Trabajo y construcción de identidades en trabajadores de la industria del Retail en Chile. Estudio en 3 ciudades sobre contextos regionales productivos, narrativas identitarias, formas de reconocimiento, control managerial y organización sindical”. 
Soto, 2015; Stecher, 2013). La pregunta por el modo en que los individuos interpretan, orientan su acción y se constituyen en sujetos laborales en el marco de contextos de trabajo marcados por procesos de modernización empresarial, flexibilización organizacional y nuevos discursos neomanageriales, ha dado lugar a una amplia producción empírica y discusión conceptual.

En términos empiricos, las investigaciones han dado cuenta, en diferentes industrias y escenarios laborales, de procesos de debilitamiento de identidades colectivas vinculadas con el mundo del trabajo industrial (identidad de clase, sindical, organizacional, oficio), de formas de construcción identitaria crecientemente individualizadas y basadas en proyectos de autorrealización personal, de nuevos discursos neomanageriales que buscan modelar la identidad laboral con base en los valores del emprendimiento y la interiorización de los valores corporativos, del tensionamiento de modelos tradicionales de género, de cambios generacionales en el vínculo subjetivo al trabajo y de un incremento de experiencias laborales vinculadas con sentimientos de precariedad, indefensión e incertidumbre (Antunes, 2003; Araujo y Martuccelli, 2012; Battistini, 2004; Dubar, 2001).

En términos conceptuales, se han producido desarrollos teóricos orientados a conceptualizar las identidades y experiencias laborales desde perspectivas más complejas que superen los reduccionismos de corte estructural -funcionalista o marxista- o psicologicista, que primaron en el análisis de las subjetividades laborales a lo largo del siglo XX (De la Garza, 2000; Du Gay, 1996; Stecher, 2013).

Se trata de marcos de análisis que procuran estudiar las experiencias e identidades de los trabajadores, reconociendo tanto la incidencia de las estructuras tecno-socio-productivas y marcos institucionales que definen los escenarios de trabajo, como la reflexividad, agencia y creación de sentido que lleva a cabo el actor laboral en el crisol de las interacciones y desafíos cotidianos de su inserción laboral. Actor laboral considerado siempre a la luz de sus trayectos biográficos, sus pertenencias colectivas dentro y fuera del trabajo, y sus posicionamientos estructurales de clase, género, generación y etnia (Battistini, 2004; Stecher, 2013).

El presente artículo busca contribuir a esta línea de investigación a partir de la presentación de los resultados de un proyecto de investigación de corte cualitativo que se propuso describir y comprender las experiencias laborales y los procesos identitarios de trabajadores/as de tienda de la industria del retail en Santiago de Chile. Específicamente, se presenta un análisis de los relatos de 31 trabajadores de tiendas por departamento (vendedores comisionistas, cajeras, asistentes de venta, jefes de departamento), orientado desde la perspectiva de la Sociología de la Experiencia (Dubet, 2010). 
El artículo describe y discute en detalle el modo en que los trabajadores, al interior de una industria fuertemente modernizada, orientan su acción a partir de las tres lógicas de la vida social (integración, estrategia y subjetivación) que distingue Dubet $(2007,2010$ y 2013) y construyen, con base en la articulación de estas, formas particulares de experiencia social en el trabajo. El análisis distingue tres modalidades o figuras de experiencia-comprometida, bloqueada y en tránsito- dentro del colectivo de trabajadores de las tiendas por departamento.

La importancia y aporte del artículo se juega en un doble plano: empírico y conceptual. En términos empíricos, aporta a la comprensión de las subjetividades y experiencias de trabajo dentro de una industria específica como es el retail. Una industria intensiva en uso de mano de obra y que, tanto en Chile como a nivel de América Latina, ha sido objeto de profundos procesos de reestructuración y modernización a partir de la concentración del mercado en grandes holding empresariales, los cuales han implementado, en el espacio de las tiendas (supermercados, tiendas por departamento, tiendas para el mejoramiento del hogar), importantes innovaciones organizativas y tecnológicas de corte flexibilizador (Calderón, 2006).

En términos conceptuales, el artículo presenta el modelo teórico de la Sociología de la Experiencia de Dubet (2007, 2010 y 2013), ilustrando, a partir de su uso en una investigación empírica, su potencial analítico para la comprensión de las experiencias laborales en el contexto de las transformaciones del trabajo. Si bien dicho modelo ha sido usado en algunas investigaciones en Chile y América Latina, su utilización ha sido más bien escasa en lo que respecta al campo de los estudios laborales. Como esperamos mostrar, el modelo de Dubet ofrece una herramienta de conceptualización y análisis fecunda para la investigación empírica sociolaboral, permitiendo desarrollar una comprensión sociopsicológica de las experiencias sociales de los trabajadores que reconoce, tanto la dimensión subjetiva de las lógicas de acción, como la dimensión estructural o sistémica de los escenarios de trabajo.

El artículo se organiza de la siguiente manera. Después de esta sección introductoria se desarrolla una sección de antecedentes que caracteriza de forma general la industria del retail y las tiendas por departamento en Chile, y una síntesis de los conceptos centrales de la Sociología de la Experiencia. Posteriormente, se presenta el diseño metodológico de la investigación. En cuarto lugar, se exponen los principales resultados del estudio, dando cuenta de cómo son desplegadas en las tiendas por departamento las lógicas de acción (integración, estrategia y subjetivación), y caracterizando luego tres figuras de experiencia social (comprometida, bloqueada y en tránsito), que 
constituyen modalidades particulares de articulación de las tres lógicas. Al final, se sintetizan y discuten brevemente los principales aportes del estudio.

\section{Antecedentes}

\section{La industria del retail en Chile}

La noción de industria del retail se utiliza para distinguir, dentro del subsector del comercio minorista, a los grandes grupos empresariales (holding) de venta masiva, uso intensivo de mano de obra y que administran grandes cadenas de supermercados, tiendas por departamento, tiendas para el mejoramiento del hogar, tiendas de fashion retail, cadenas de farmacia u otros locales especializados (Stecher, 2012). En el caso de Chile, al igual que a nivel global, dicha industria experimentó un fuerte proceso de restructuración productiva y modernización empresarial desde los años ochenta (Calderón, 2006; Du Gay, 1996; Wrigley y Lowe, 2010).

Dicho proceso se caracterizó por una fuerte inversión de capital y apertura de tiendas a lo largo de todo el país, una fuerte concentración del mercado en unas pocas empresas, la incorporación de profundas innovaciones tecnológicas y de gestión (lean retailing), y la profesionalización, estandarización y racionalización de procesos en toda la cadena del modelo de negocio (Calderón, 2006; Gálvez et al., 2009). En Chile la industria del retail emplea alrededor de 500.000 asalariados (Fundación Sol, 2016), destacándose siete grandes grupos empresariales: Cencosud, Falabella, La Polar, Ripley, WalMart-Chile, SMU, ABCDIN.

$\mathrm{Al}$ igual que las grandes corporaciones multinacionales del retail (Walmart, Carrefour, etc.), los holding chilenos más importantes se han expandido por distintos países de América Latina, son parte de redes globales de diseño, fabricación y suministro de productos, gestionan simultáneamente distintas cadenas comerciales y formatos de tienda, articulan el negocio de la venta con otros nichos de rentabilidad (retail financiero, negocio inmobiliario, marcas propias, ventas de seguros, etc.) y han jugado un rol central -a partir de la publicidad, la expansión de centros comerciales y la entrega de tarjetas de crédito- en la expansión de las prácticas e imaginarios de consumo que definen la modernidad contemporánea (Stecher, 2012).

Respecto al formato específico de las tiendas por departamento, es posible señalar que se trata de establecimientos comerciales organizados en distintos pisos de venta y departamentos (vestuario, deportes, juguetería, computación, electrodomésticos, línea blanca, etc.) y que ofrecen una variedad de bienes 
de consumo durables. Las tiendas funcionan como unidades de negocio independiente y cada una es administrada por un gerente de tienda, a quien reportan los gerentes de piso a cargo de un conjunto de departamentos. Los departamentos, a su vez, son administrados por los jefes de departamento, a cargo de un equipo de trabajadores de primera línea (vendedores, cajeras, asistentes de venta), con el cual debe cumplir los objetivos de venta mensual. Las tiendas tienen entre 200 y 500 trabajadores, dependiendo de los metros cuadrados y del volumen de ventas; en 2015 las tres empresas más importantes en Chile (Falabella, Ripley, Paris) empleaban en total a 49.253 trabajadores.

$\mathrm{Al}$ igual que los otros formatos del retail, las tiendas por departamento emplean predominantemente a trabajadoras mujeres, jóvenes, de baja calificación, con escasa experiencia laboral previa y pertenecientes a sectores urbano-populares (Stecher, 2012). Se trata de una fuerza de trabajo con altas tasas de rotación y que recibe ingresos promedios entre 1,5 y 2,5 sueldos mínimos (Fundación Sol, 2016). A pesar de las altas tasas de rotación, existe un porcentaje importante de trabajadores (alrededor del 15\%) que cuenta con más de 10 años de antigüedad y han hecho carrera dentro de las empresas.

En términos de las relaciones laborales, se trata de empresas con una alta tasa de sindicalización (sobre el 50\%), una fuerte asimetría en la relación empresa-trabajadores, y una fuerte orientación gerencial hacia la individualización de la relación laboral. Respecto a las estrategias de gestión y al proceso de trabajo, se observa el uso intensivo de diversas estrategias flexibilizadoras: formas contractuales atípicas (especialmente contratos temporales en temporada alta); jornadas horarias flexibles; sistemas de salario variables; externalización de funciones; polifuncionalidad; reducción de tiempos muertos e intensificación de la carga de trabajo; fuerte demanda de trabajo emocional; mecanismos de control normativos basados en la promoción de la cultura corporativa; sistemas de evaluación permanente del desempeño; fuerte orientación a la calidad del servicio; programas de capacitación; entre otras (Gálvez et al., 2009; Stecher et al., 2010).

Dichas estrategias coexisten con principios de corte (neo)taylorista-fordista: estandarización y protocolización del proceso de trabajo (en especial en el caso de cajeras y asistentes de venta); organización fuertemente jerárquica y piramidal; baja participación de los trabajadores en la toma de decisiones y presencia de mecanismos de vigilancia y control directo (Stecher et al., 2010). 


\section{La Sociología de la Experiencia}

El análisis de los relatos de los trabajadores y la reconstrucción de su experiencia laboral en las tiendas se llevó a cabo a través de la Sociología de la Experiencia de Dubet. Dicha perspectiva se inscribe en el campo más amplio de las Sociologías del Individuo (Martuccelli y De Singly, 2012), un programa de investigación que parte del diagnóstico de que la vida social contemporánea ya no puede ser comprendida desde los operadores analíticos clásicos que la sociología generó para analizar la sociedad industrial del Estado-Nación (Dubet, 2007; Martuccelli, 2007). Estos operadores consisten en, por un lado, la idea de sociedad, entendida como un sistema estable y coherente, y la idea del personaje social, por otro, que refiere a la correspondencia entre la posición del actor en la estructura social y los valores, las normas y las identidades que este interioriza.

Ante esa crisis y agotamiento de la imagen de "la sociedad" como orden funcional en que existe una adecuación entre el sistema y el actor, las sociologías del individuo proponen estudiar la vida social contemporánea, partiendo del análisis de las experiencias individuales y del trabajo de construcción de sí mismo que llevan a cabo los actores (Dubet, 2013). No se trata, en ningún caso, de renunciar al análisis de los procesos y mecanismos sociales, sino de reconstruirlos desde el nivel de las experiencias, las lógicas de acción y las interacciones de los individuos (Dubet, 2013; Martucelli, 2007).

En ese marco Dubet $(2007: 117)$ propone analizar la vida social a partir de la noción de experiencia social, entendiendo por esta "la cristalización, más o menos estable, en los individuos y los grupos, de lógicas de acción diferentes, a veces opuestas, que los actores deben combinar y jerarquizar a fin de constituirse como sujetos". La propuesta asume que los individuos están confrontados permanentemente a la tarea de articular distintas lógicas de acción para construir una experiencia consistente y con sentido, y configurarse como autores de la misma (Martucelli y De Singly, 2012).

Se señala, a su vez, que en el contexto de las transformaciones de las sociedades contemporáneas - globalización, individualización, pluralización de mundos de vida - las lógicas de acción se disocian, tensionan y no responden a un principio articulador común, recayendo en los propios individuos la tarea de dar forma y consistencia a la experiencia en el proceso continuo de enfrentar y resolver los desafíos de la vida social.

Dubet va a distinguir tres grandes lógicas de acción en la vida social: la integración, la estrategia y la subjetivación. Cada lógica de acción remite a subsistemas que la anteceden, expresa una cierta forma de atribución de 
sentido, define parámetros específicos de legitimidad de la acción, da cuenta de modos determinados de representar y posicionarse como actor en la vida social, y constituye un particular modo de definición de sí y de vínculo a los otros (Dubet, 2013).

\section{La lógica de la integración}

La lógica de la integración da cuenta de aquellos comportamientos y pensamientos que proceden de la interiorización de las identidades, normas y modelos culturales que ofrece la sociedad en general, y los específicos colectivos de referencia y pertenencia en particular. Desde esta lógica el actor ve la sociedad como un sistema de integración, en el cual ocupa un rol y sostiene una identidad que define el modo adecuado de actuar. Desde esta perspectiva, el actor orienta y justifica su acción apelando a pertenencias colectivas ("nosotros los vendedores", "nosotras las mujeres jefas de hogar", etc.), a normas, mandatos, roles y valores sociales internalizados ("ser un buen trabajador", "ser un buen padre"). En esta lógica opera como un mecanismo clave la producción de diferencias entre "nosotros" versus "ellos", a partir del cual se define y sostiene la propia posición e identidad en la sociedad, se diferencian grupos y colectivos, y se busca la afirmación y estabilidad del orden social (Dubet, 2007, 2010 y 2013).

\section{La lógica de la estrategia}

En esta lógica, el actor se representa la vida social como un mercado o campo de competencia, donde cada individuo es un estratega que moviliza distintos medios y recursos para conseguir sus objetivos. Acá los comportamientos y pensamientos son justificados en función de los intereses que cada actor persigue, los cuales pueden ser consistentes o no con los valores colectivos propios de la integración. Los intereses pueden ser múltiples, cambiantes, y dan lugar a la realización de cálculos y a la definición de estrategias al interior de las reglas de juego específicas de los distintos dominios sociales.

En este plano, los actores movilizan sus identidades sociales no como una expresión de pertenencia, solidaridad y tradición, sino como recursos estratégicos con los que se busca convencer, manipular o presionar a otros para obtener ciertos resultados e incrementar el propio poder. Aquí la diferenciación entre colectivos no se da en los términos de nosotros/ellos, sino en términos de aliados y competidores, cambiantes y contingentes, en las redes 
locales de las distintas interacciones en las cuales el actor participa (Dubet, 2007, 2010 y 2013).

\section{La lógica de la subjetivación}

Esta lógica da cuenta de aquella perspectiva por la cual todo actor se piensa como un individuo singular y crítico, que no se reduce a sus pertenencias colectivas ni a sus acciones estratégicas, y que es capaz de tomar una distancia reflexiva respecto a la sociedad y a sí mismo. Desde esta perspectiva, la sociedad no es un sistema de integración ni un mercado o campo de competencia, es un sistema distante y distinto al individuo que opera como fuente de dominación y bloqueo a los potenciales y deseos de autorrealización y autenticidad de los actores.

En esta lógica, los comportamientos y pensamiento son justificados en tanto expresiones de (o intentos de expresar) la autonomía, las convicciones morales, la vocación, la perspectiva singular e irreductible de cada individuo, frente a los mecanismos sistémicos de producción y control social. Por lo general, esta lógica de la subjetivación se expresa en términos negativos, ahí donde el individuo reconoce y se distancia críticamente del sistema y las instituciones, o ahí donde padece encarnadamente aquellas fuerzas o situaciones que lo oprimen y limitan su deseo y potencial de ser sujeto (Dubet, 2007, 2010, 2013).

El mundo del trabajo es una esfera de la vida social donde es posible reconocer con claridad el despliegue de estas tres lógicas por parte de los trabajadores (Dubet, 2006). Por un lado (integración), el trabajo, en especial en su modalidad de empleo asalariado, es un mecanismo central de socialización e integración, de acceso a colectivos de pertenencia e identificaciones (oficio, clase, empresa), de inclusión en redes de protecciones y derechos que confieren a los individuos un lugar y un estatus legitimado en el orden social. A su vez (estrategia), el trabajo es un intercambio mercantil donde el empleado arrienda su fuerza de trabajo a cambio de un salario, y procura estratégicamente mejorar, o al menos mantener, a través de negociaciones individuales o acciones colectivas (sindicatos), las condiciones de dicho intercambio (renta, movilidad, beneficios, condiciones materiales, etc.). Por último (subjetivación), el trabajo es para los actores un espacio que permite potencialmente expresar la vocación y afirmar la autonomía y singularidad, al mismo tiempo que un ámbito fundamental en las experiencias subjetivas de control, dominación y sufrimiento que vivencian los actores frente a los imperativos productivos y sistémicos de la vida social moderna. 


\section{Diseño metodológico}

Se utilizó una metodología cualitativa basada en entrevistas a trabajadores de las tiendas por departamento en Santiago de Chile, las cuales estuvieron centradas en su experiencia laboral y fueron analizadas siguiendo los procedimientos de la grounded theory (Strauss y Corbin, 2002).

\section{Participantes}

El estudio se enfocó tanto en empleados de primera línea como en jefaturas directas. En ambos casos se trató de personal directamente contratado por la empresa, de manera indefinida y a jornada completa. Participaron 31 trabajadores, de los cuales 25 eran de primera línea (vendedores comisionistas, asistentes de venta y cajeros) y seis jefaturas: 19 mujeres y 12 hombres. El promedio de edad fue 39 años con un rango entre 22 y 61 años. El tiempo promedio en la empresa fue de seis años, registrándose una variación desde los cuatro meses hasta los 24 años.

Complementariamente, se consideró la participación de seis expertos de la industria en calidad de informantes claves: gerente de tiendas, dirigentes sindicales, encargados de recursos humanos e investigadores.

\section{Producción de información}

El estudio consideró el uso de dos modalidades distintas de entrevista. Por un lado, con los trabajadores de tienda y con el objetivo de acceder al despliegue de orientaciones de acción de los trabajadores y a la construcción de su experiencia laboral en las tiendas, se llevaron a cabo entrevistas semidirectivas (Martuccelli y de Singly, 2012), una modalidad caracterizada por otorgarle un papel central a la reflexividad de los individuos durante la conversación.

En este contexto, el dispositivo de entrevista buscó acentuar el trabajo sobre sí mismo de los trabajadores, estimulando la capacidad reflexiva mediante preguntas orientadas a la comprensión de las acciones desplegadas y los significados otorgados en los distintos ámbitos del trabajo. Si bien se ahondó en las trayectorias laborales previas de los trabajadores y las narrativas y sentidos asociadas a las mismas, las entrevistas abordaron también la complejidad del "aquí y ahora", orientando las preguntas hacia el sentido de las acciones de los trabajadores en el presente, para procurar el retorno reflexivo sobre las circunstancias actuales de su experiencia laboral (Martuccelli y de Singly, 2012). 
Por otro lado, con los informantes claves se utilizó la entrevista semiestructurada (Flick, 2004), la cual estuvo dirigida a recoger información sobre las tiendas por departamento como escenario laboral y sus transformaciones recientes.

\section{Procedimiento}

Las entrevistas fueron realizadas durante los años 2014 y 2015 por parte del equipo de investigación en lugares próximos al lugar de trabajo. El acceso al campo consideró diversas modalidades para concertar las entrevistas, entre las cuales cabe mencionar el contacto directo de los trabajadores durante la jornada laboral, el vínculo con sindicatos y la estrategia "bola de nieve". En el caso de los informantes claves, se efectuó un contacto directo vía correo electrónico.

En todos los casos se consideró la firma de un consentimiento informado, que explicitó los objetivos del estudio y garantizó confidencialidad y anonimato. La duración promedio de las entrevistas fue de 90 minutos, siendo grabadas y transcritas para el análisis.

\section{Análisis}

Se utilizaron los procedimientos de codificación abierta y axial de la grounded theory (Strauss y Corbin, 2002). En una primera etapa de codificación "abierta", los datos fueron interrogados para abrir los significados vertidos en las entrevistas, generando códigos sencillos y concretos para relacionarlos a cada una de las tres lógicas de la experiencia social (integración, estrategia y subjetivación). En una segunda etapa, se llevó a cabo la codificación "axial” en la cual se relacionaron los códigos previamente generados y se elaboró una comprensión de mayor densidad y complejidad de las lógicas de acción. El análisis evidenció, mediante un ejercicio de comparación constante, declinaciones específicas de las lógicas de acción en función de las características de los trabajadores (sexo, edad, antigüedad y puesto). A partir de ello fue posible construir tres figuras de experiencia laboral en las tiendas. Respecto a las entrevistas a informantes claves, estas fueron analizadas con los mismos procedimientos de codificación, pero con un foco en la descripción de las tiendas como escenario laboral. 


\section{Resultados}

\section{Las lógicas de acción en las tiendas por departamento}

\section{Integración}

Esta lógica se encuentra determinada fuertemente por el momento de ingreso a trabajar en la tienda. Este es vivido como una experiencia que marca un antes y un después, y que implica adquirir el rol de trabajador asalariado y empleado de una gran empresa. En ese sentido, en el corazón de esta lógica se encuentra la configuración de una "promesa" de integración, a nivel del mercado laboral y de la propia empresa, que resulta hasta cierto punto atípica a la luz del perfil de la mano de obra: bajo nivel de calificación, falta de experiencia laboral, trayectorias laborales discontinuas y en muchos casos en el sector informal.

La situación formal de empleo en las empresas (contrato indefinido, jornada completa, remuneración mensual estable, beneficios, protecciones sociales, posibilidades de capacitación, oferta de movilidad interna, participación en sindicato y negociaciones colectivas, etc.) contrasta con las trayectorias laborales previas de muchos de los trabajadores del retail, caracterizadas por recorridos laborales desarticulados y discontinuos que se distancian del ideal de una carrera laboral ascendente y protegida. Como señala una de las entrevistadas: "lo bueno, la estabilidad en el sueldo y la estabilidad en las fechas de pago, que es súper importante. Mi contrato decía que me pagaban el último día del mes y sagradamente el último día del mes me pagaban, siempre" (Vendedora, 57 años). ${ }^{2}$

Como lo han documentado estudios en Chile (Araujo y Martuccelli, 2012; Todaro y Yáñez, 2004), las experiencias laborales de segmentos importantes del mercado laboral en Chile se caracterizan por la "pluriactividad", la diversidad de oficios y actividades realizadas tanto a lo largo de una trayectoria (diacrónica) como también en un mismo momento de esta (sincrónica); por "recorridos inerciales" basados más en desplazamientos horizontales y el aprovechamiento de situaciones contingentes, que en la planificación de un proyecto de carrera ascendente; y por ciclos -especialmente en las mujeresde entrada y salida del mercado laboral en función del cuidado de los hijos y labores domésticas.

En ese sentido, el ingreso a las empresas del retail homogeniza en un estatuto común de empleo la pluralidad e inestabilidad de las trayectorias

2 Entrevista personal, 21 de abril 2014, Santiago, Chile. 
laborales y biográficas previas, y opera como un clivaje entre las experiencias laborales anteriores y un modo de inserción asalariada, de mayor estabilidad, que crea o refuerza en un colectivo diverso de trabajadores el rol e identidad de empleados de grandes empresas del sector comercio. Rol socialmente legitimado y normativamente regulado desde el cual orientan su acción, demandan derechos y asumen tareas y responsabilidades en el trabajo en las tiendas.

Siempre fueron trabajos esporádicos, así como te digo, en temporada de navidad, [...] también trabajé en una empresa de marketing y de eso me llamaban de vez en cuando, que era de telefonista. Mucho tiempo antes de que yo me embarazara del primer niño trabajé también en un jardín particular, era cocinera, siempre trabajé en nada de lo que yo estudié [...]. Cuando yo recién me separé, yo obviamente tenía que trabajar en lo que fuese porque necesitaba plata y me puse a trabajar en casa particular haciendo aseo, porque era lo que a mí me generaba más plata [...] No importa, le dije yo [en la entrevista de ingreso a la tienda], no me importa ganar poco menos acá [...] porque a mí me gusta trabajar en retail [...] yo no quería seguir trabajando en casa (Asistente de venta, mujer, 34 años). ${ }^{3}$

En segundo lugar, esta lógica se vincula con las condiciones de sociabilidad en las tiendas y al lugar simbólico del retail en los imaginarios de la sociedad de consumo. El trabajo dentro de las tiendas supone la participación en una sociabilidad cotidiana, dada por las exigencias funcionales de la coordinación del trabajo de venta, y por los espacios de sociabilidad informal e interpersonal que se generan durante la jornada (Stecher et al., 2010). La tienda es concebida como un ambiente laboral, no sin tensiones mediante, que ha ampliado los círculos sociales y en donde suelen predominar vínculos horizontales y modalidades -especialmente a nivel de los colegas cercanos, clientes conocidos y jefaturas directas- de apoyo y reconocimiento recíproco.

La apertura de espacios de sociabilidad opera también para los trabajadores en un plano simbólico más amplio, vinculado con la generación de mayores posibilidades de participación en prácticas sociales, interacciones y espacios urbanos (la gran empresa, el mall, los dispositivos tecnológicos de las tiendas) que expresan imaginarios globales de consumo, progreso y modernización.

En tercer lugar, el trabajo en las tiendas es reconocido como una posibilidad de aprendizajes, vía capacitaciones formales o vía el ejercicio cotidiano del cargo. La experiencia laboral conlleva el aprendizaje de un conjunto de funciones y destrezas específicas (dominio de caja, reposición de mercadería, realización de inventarios, atención al cliente, etc.), así como de los productos y las marcas que la tienda ofrece (ropa, calzado, dispositivos electrónicos, etc.). Dentro de estas, un lugar especial lo tienen las llamadas habilidades

3 Entrevista personal, 13 de octubre 2014, Santiago, Chile. 
"blandas", vinculadas al trabajo emocional que se despliega en la atención al cliente, y el que exige una autorregulación de las actitudes y comportamientos de los vendedores. En los trabajadores con largos años de experiencia en la industria, por su parte, las tiendas por departamento ofrecen la oportunidad de construir y desarrollar verdaderas identidades de oficio relacionadas al comercio y las ventas, en torno a las cuales se construyen fuertes sentido de pertenencia que orientan la acción.

En cuarto y último lugar, el análisis de las entrevistas indica que la lógica de la integración se vincula con la promesa que hace la empresa, especialmente al inicio, de desarrollar una carrera dentro de la organización, con base en principios de mérito y a partir de la oferta simbólica de identificarse con las exigencias y valores corporativos. En efecto, los trabajadores reconocen que constantemente aparecen ofertas laborales en la empresa y que las jefaturas no solo suelen mencionar la existencia de oportunidades en la tienda, sino que ellas mismas se presentan como testimonio de las posibilidades de promoción interna. Un asistente de venta cuenta que al ingresar "el jefe me dijo 'mira, acá está la opción' me dijo, 'tú podí entrar, no sé po, haciendo aseo ahí en [la empresa] y podí terminar como yo... y eso no va a ser en un lapso de 10 años. Si en un año o dos años lo hiciste bien, te evalúan acá...” (Asistente, hombre, 28 años). ${ }^{4}$

\section{Estrategia}

Esta lógica da cuenta de formas de acción orientadas por el cálculo y la búsqueda estratégica de mantener la posición laboral alcanzada, maximizar los beneficios que ofrece el empleo en las tiendas (bonos, comisiones) y, en algunos casos, proyectar - a partir del desempeño, los contactos y la mejora de la empleabilidad- posibilidades de movilidad en la organización o en el mercado laboral.

En general, predomina, en especial en los cargos de menor calificación y responsabilidad (cajeras, asistentes de venta), una estrategia sobre todo defensiva: una disposición que antes que orientada a avanzar laboralmente y diseñar estrategias de largo plazo, intentaría sostener la posición actual de empleo, arreglárselas en el día a día asegurando el ingreso mensual y no retroceder en las condiciones alcanzadas.

No obstante, si la orientación estratégica en el registro del proyecto laboral es en especial defensiva y presentista, en la cotidianidad de la tienda

4 Entrevista personal, 20 de enero 2015, Santiago, Chile. 
alcanza una expresión algo más amplia y definida, como consecuencia de las estrategias gerenciales. El proceso de trabajo y la gestión de recursos humanos es un elemento detalladamente planificado por los holding del retail, procurando reducir costos laborales, incrementar la productividad y extraer las máximas ganancias, en específico mediante modelos de atención al cliente, que se basan en regímenes de remuneración variable en función de distintos incentivos económicos.

Por un lado, el modelo de atención personalizado funciona con base en la figura del vendedor comisionista que se encuentra en los departamentos de productos más complejos y especializados (muebles, línea blanca, informática). Bajo este modelo, la organización del trabajo se concibe como un mercado en donde las comisiones de ventas representan el capital en juego, y los vendedores que concurren a este y compiten individualmente por maximizar sus utilidades.

Los vendedores comisionistas, en ese sentido, deben desplegar de forma permanente una racionalidad estratégica en el día a día, pues de ello depende el sueldo que obtendrán a fin de mes. Por esta razón, aunque los vendedores valoran a sus compañeros, al mismo tiempo saben que son competidores, lo cual los condiciona a permanecer en un estado de alerta que a veces genera conflictos entre ellos.

Por otro lado se encuentra el modelo de venta asistida (asistente de venta y cajeros), asociado a departamentos de la tienda (vestuario, juguetería, niños), donde no resulta indispensable la presencia de un vendedor especializado, y en el cual los incentivos económicos se organizan con base en bonos -y no comisiones como porcentaje de venta- de carácter colectivo (asociados al cumplimiento de metas de venta y por indicadores de satisfacción al cliente) e individuales (relacionados con la asistencia y la puntualidad en el trabajo).

Por ello la racionalidad estratégica se ve menos estimulada, pues con la eliminación de las comisiones individuales el ingreso tiene un límite fijo e inferior al de un vendedor comisionista. Además, hace menos directa la consecución de los incentivos, porque ya no dependen del trabajador individual, sino de todo el equipo del departamento. Con todo, si bien se reduce el espacio de la estrategia, no lo elimina, pues -como sugiere el siguiente extracto- el sueldo sigue dependiendo en alguna medida del desempeño.

Yo soy preguntona, yo, no sé, si me puedo hacer cargo de... “¿cuánta es la meta de este mes?” o “¿cuánta era la meta de esta semana?” o “¡cómo vamos?” Yo soy así porque a mí sí me importa cuánto gano, $[\ldots]$ necesito saber si este mes me va alcanzar pa cubrir todo lo que yo tengo que cubrir (Asistente, mujer, 22 años). ${ }^{5}$

5 Entrevista personal, 7 de diciembre 2014, Santiago, Chile. 
Otro eje de la lógica de la estrategia se relaciona con las acciones desplegadas por los trabajadores, para hacer frente y aminorar las consecuencias negativas de un conjunto de innovaciones organizativas implementadas por las empresas en los últimos años. Se trata de medidas que apuntan a reducir costos a partir de la disminución de personal (vía automatización y sistemas de autoatención), y el reemplazo de trabajadores antiguos y con mejor remuneración por un nuevo perfil de trabajador joven, con menor remuneración e inserto en puestos que implican la descualificación del trabajo de venta y el socavamiento de identidades de oficio del sector (Stecher y Martinic, 2018). Aquello genera una actitud vigilante, atenta y sospechosa ante los cambios en la gestión que ponen en práctica las empresas del retail.

Sabemos [...] que viene un contrato nuevo para el área nuestra y yo no lo voy a firmar. Porque prefiero a que me despidan con lo que yo tengo y gano en este momento [...] y creo que ya en el retail no existen [esos contratos antiguos], porque creo que lo único que quieren es llegar a un horario con un sueldo mínimo... Los retail yo creo que unos dos o tres años más no va haber alguien que te atienda en un retail, va ser el autoservicio (Vendedora, 59 años). ${ }^{6}$

Por otro lado, cabe mencionar que los sindicatos, en tanto mecanismo colectivo de acción estratégica, tienen un carácter ambivalente. Por un lado, poseen una alta tasa de afiliación en las tiendas por departamento, y los dirigentes son conocidos y juegan un rol importante en las tiendas a la hora de informar los beneficios, interceder ante situaciones puntuales, acompañar procesos de denuncia ante la Dirección del Trabajo y organizar los procesos de negociación colectiva. Con todo, el poder y la fuerza estratégica del colectivo sindical como actor sociopolítico, capaz de articular masivamente a los trabajadores y lograr una mayor redistribución del poder y los beneficios dentro de la empresa, es aún débil. Ello se debe, entre otros factores, a la imposibilidad de la ley laboral en Chile para la negociación colectiva por rama, la alta heterogeneidad y rotación de la fuerza de trabajo, las orientaciones culturales más individualizadas, el gran poder económico de los holding y el vínculo instrumental de muchos trabajadores hacia el sindicato.

Por último, cabe señalar que algunos de los trabajadores entrevistados despliegan una lógica de acción estratégica que, más allá de lo antes señalado, se orienta en un horizonte temporal más amplio y con mayores niveles de planificación a la luz de objetivos de movilidad ascendente en la organización o el mercado laboral.

6 Entrevista personal, 29 de marzo 2015, Santiago, Chile. 


\section{Subjetivación}

En la subjetivación se abre un dominio en el cual la acción del trabajador, sus modos de comportamiento y representación en el espacio de la tienda va más allá del rol de asalariado o empleado; así como más allá también de la acción estratégica. Se trata de una orientación con una multiplicidad de registros, en ocasiones ambivalentes e incluso contradictorios, que abarcan los sentidos de realización personal en el trabajo, los padecimientos de la dominación e injusticias laborales, y la capacidad de distanciamiento y análisis crítico respecto a la empresa y el trabajo.

En primer lugar se encuentran referencias a experiencias significativas, satisfactorias y singulares de realización personal en el trabajo en las tiendas. Por un lado, se observa un móvil de realización vinculado al trabajo y al conjunto de actividades que implica, en el cual se disfruta por una amplia gama de razones: por su intensidad y sensación constante de estar en movimiento; porque es lúdico, entretenido y se pasa bien; porque ha permitido el descubrimiento de las capacidades individuales y un sentido de utilidad o porque es el espacio en donde se despliega una experticia o saber de oficio. Por otro lado, otro móvil de realización se construye en torno a las relaciones sociales, a los compañeros, los jefes e incluso a los clientes, constituyendo uno de los principales soportes existenciales de los trabajadores que hacen más llevadero el trabajo y la vida misma.

Pero la subjetivación, en segundo lugar, es también una lógica que remite a los sufrimientos y padecimientos en el trabajo, y a la frustración por las expectativas personales o promesas organizacionales no cumplidas. Sin duda, una de las principales fuentes de sufrimiento se relaciona con la demanda temporal asociada a jornadas laborales de 11:00 a 21:00 horas y a un sistema de turnos de $5 \times 2$. Los trabajadores sienten que pierden el día en la tienda, que salen tarde en relación con el "horario de oficina" y llegan todavía más tarde al hogar, cuando sus hijos están durmiendo. Además, como los turnos varían permanentemente y laboran los fines de semana, suelen no estar presente para instancias de reunión familiar como celebraciones, cumpleaños o Navidad. Sin embargo, aunque el padecimiento afecta tanto a hombres como mujeres, son ellas las que más lo resienten: tanto en el plano emocional por el distanciamiento con sus hijos, y como cansancio físico y mental por la doble jornada laboral que implica el cuidado del hogar.

El retail es duro y es pesado. Es complicado, porque no tienes calidad de familia, porque tú pierdes todo. No tienes calidad de familia, o sea muchas veces tú sales de tu casa y tu 
hijo se fue al colegio y cuando vuelves tu hijo está durmiendo. Y los días libres, entre comillas, te sirven para hacer aseo y hacer todo, para organizar tu casa, y te quedan los pocos momentos (Vendedora, 34 años).?

Respecto a las jornadas de trabajo (extensas, variables y a-típicas), pero en un sentido más amplio, los entrevistados reportan experiencias de sufrimiento vinculadas al cansancio físico y emocional que supone trabajar de pie, regulando las propias emociones en las interacciones con clientes, teniendo en mente las continuas exigencias de logro de metas, y en un contexto de intensificación del trabajo por la reducción de trabajadores.

En el retail trabajamos con el tema de la garantía, si tú no vendes garantía, te ponen el peso todo el rato: "vende eso, vende eso". Eso es lo primordial. Si vendes un producto tiene que ir con eso, te presionan, [...] A mí me dio ansiedad y trastorno del sueño por trabajar tanto en la tienda. De hecho, también tenía compañeros que también estaban con licencia psiquiátrica. Tengo una compañera que está con medicamentos, porque no puede dormir. La presión que te hacen todo el día es algo que te deja bastante loco (Vendedor, 25 años). ${ }^{8}$

Asimismo, el tema de los bajos sueldos para aquellos trabajadores con responsabilidades familiares y que no cuentan con comisiones individuales por venta, en el contexto de empresas con grandes niveles de ganancia aparece también como un motivo de insatisfacción asociado a una experiencia de injusticia.

Otra fuente de padecimientos se relaciona con el maltrato que han sufrido algunos trabajadores por parte de los clientes. Se trata de experiencias de abuso, humillación, violencia verbal y psicológica, donde los clientes buscan demostrar una superioridad de clase y de género (pues suele ser ejercida sobre mujeres) frente al trabajador de primera línea. Como señala un entrevistada: "Hay gente que te puede tirar el título encima diciendo que tú eres cualquier cosa o que tú por ellos ganas plata [...] hay gente que te dice que porque ellos compran en, tú ganas tu sueldo [...] 'Yo te estoy pagando', es así' (Asistente de ventas, mujer, 25 años).?

Finalmente, la lógica de la subjetivación se expresa en el modo en que los trabajadores establecen una distancia crítica respecto a la empresa, afirmando, frente a las demandas sistémicas de la industria, una perspectiva singular de reflexividad desde la cual también orientan y dotan de sentido su acción en las tiendas. Desde los trabajadores más antiguos y con fuertes identidades de oficio, por ejemplo, se tiene una visión crítica de las reorganizaciones recien-

7 Entrevista personal, 14 de agosto 2014, Santiago, Chile.

8 Entrevista personal, 7 de diciembre 2014, Santiago, Chile.

9 Entrevista personal, 2 de octubre 2014, Santiago, Chile. 
tes llevadas a cabo dentro de las tiendas, las que, como señalamos, han estado orientadas a buscar reducir costos y por la descualificación del trabajo, y se han implementado sin consultar a los trabajadores, lo cual ha implicado una cosificación de estos.

De acuerdo con un testimonio: "Ha dejado de ser la empresa familiar que era antigua, que había sido, y que trataba al empleado... ¡como empleado! O sea, lo conocía, no era un número para la empresa, sino era alguien que valía, con que se podía conversar y todo" (Vendedora, 57 años). ${ }^{10}$

\section{Figuras de la experiencia social}

Junto con la reconstrucción de las lógicas de acción, el análisis identificó las siguientes tres figuras de experiencia social en el trabajo de las tiendas, las cuales emergen a partir de singulares formas de articulación de la integración, la estrategia y la subjetivación. Cada figura da cuenta de una modalidad particular de construcción de sentido y orientación de la acción dentro de las tiendas por departamento y se vincula con ciertas trayectorias y/o colectivos específicos de trabajadores.

\section{La experiencia comprometida}

En este tipo de experiencia existe una fuerte relación de pertenencia con la empresa o el rubro; se desarrolla una identificación con el trabajo efectuado, concibiéndose este además como un móvil de realización personal; las proyecciones se relacionan con continuar trabajando en el oficio o en hacer carrera en la organización. Se trata, así, de una experiencia caracterizada por un fuerte compromiso e implicación subjetiva con el trabajo en las tiendas, que si bien no exime a los trabajadores de los padecimientos propios del trabajo en el retail, estos aparecen en un segundo plano respecto a la valoración de la trayectoria laboral y de la realización personal alcanzada, y se asumen como parte de las reglas del juego que rigen las condiciones laborales del sector.

Los sentidos de pertenencia (integración) que se generan aquí van más allá del mero rol de asalariado y se extienden a la identificación con los valores de la empresa y/o con un oficio valorado por la organización. Por ello, se trata del caso en que la socialización en la organización consigue su mayor grado de éxito, pues la experiencia laboral se constituye teniendo a esta como referencia principal, en gran medida gracias a la profesionalización que han significado las capacitaciones que brindan tanto empresas como marcas de 10 Entrevista personal, 21 de abril 2014, Santiago, Chile. 
productos, el desarrollo de competencias en el ejercicio mismo de los cargos y la acumulación de experiencia a través de los años en el rubro.

En los vendedores identificados con su oficio, los aspectos fundamentales de la socialización se relacionan con la especialización en los productos de un área y en un conocimiento técnico sobre estos, así como en una atención al cliente en la que se es experto y donde quien lleva el control de las ventas es el trabajador. En el caso de las jefaturas intermedias de departamento, en donde además suele existir una alineación con los valores corporativos, la identificación con el oficio se da en el plano de la gestión de un departamento, del "saber hacer" de un jefe. En efecto, la gestión constituye una dimensión prevalente que da cuenta de eficacia operativa, sentido de competencia y dominio sobre el trabajo que revelan la adquisición de un sentido práctico (gestionar equipos, ofertas, clientes, metas, etc.), que es clave para el funcionamiento cotidiano de las tiendas.

A su vez, en esta forma de experiencia laboral "comprometida" predomina un mayor conocimiento de las oportunidades de desarrollo en la empresa, una orientación a aprovecharlas en una perspectiva de largo plazo y con mayores expectativas en cuanto al desarrollo profesional. La estrategia, así, ha dado lugar a un proyecto basado en la construcción de una carrera en la empresa o rubro, lo cual es cierto incluso para el caso de los trabajadores de primera línea con identidades de oficio que no han ascendido.

En tal caso, como el ejercicio del oficio de vendedor tiene un importante grado de acople al régimen de remuneración en función de comisiones - pues lo potencia y lo incentiva-, el proyecto consiste en continuar bajo esas condiciones que ya han posibilitado el acceso a una situación estable para el trabajador y su familia, y seguir sacándoles el máximo provecho.

Entre quienes llegaron a ser jefes, por su parte, se observa cómo les ayudó el mantenimiento de buenas relaciones con compañeros y superiores, y la disposición a asumir diferentes tareas, mostrarse siempre disponible y comprometerse con la empresa sin prestar resistencia. Dan cuenta del aprovechamiento de las oportunidades de promoción que aparecen en el trabajo, con un sentido de garantizar o aumentar el ingreso, pero incentivados también por el reconocimiento de la empresa a sus aptitudes.

En general, esta forma de experiencia suele enfatizar el "mérito propio" y una orientación en la vida individualista, donde los logros son el resultado de esfuerzos y sacrificios personales. En ese sentido, el trabajo en la tienda ha sido la base para consolidar un proyecto personal, pero también familiar, en el cual se destaca luchar por la educación de los hijos y el acceso a un nivel de 
bienestar mejor. "Yo todas las cosas que yo tengo, mi casa, se lo debo a Ripley, a mi trabajo y a Ripley" (Vendedora, 59 años). ${ }^{11}$

Cuando aparece una distancia crítica, esta se refiere fundamentalmente a las re-organizaciones recientes de las empresas del retail, que han implicado la reducción de los vendedores con mayor antigüedad, experiencia y remuneración, y su reemplazo por trabajadores jóvenes, sin experiencia ni compromiso, con alta rotación, e insertos en procesos de trabajo de venta fuertemente protocolizados. Se critican esos cambios en las empresas, así como el poco compromiso y la baja valoración que le otorgan al trabajo en la tienda las generaciones más jóvenes, todo lo cual aparece cuestionando y amenazando las propias trayectorias e identidades laborales. En las jefaturas intermedias, además, se añaden las dificultades y desafíos al ejercicio de la autoridad que supone dirigir y coordinar en el proceso de venta trabajadores jóvenes, menos implicados y que no se proyectan en el sector.

Esta forma de experiencia comprometida se asocia sobre todo, pero no exclusivamente, a los trabajadores con mayor antigüedad, vinculados a puestos de mayor autonomía y reconocimiento simbólico y material (jefaturas de departamento, vendedores comisionistas especializados), que han hecho una carrera dentro de la industria, la que se puede expresar tanto en la construcción de identidades laborales vinculadas al oficio de venta y/o a la pertenencia a la organización, como en los procesos de promoción y desarrollo dentro de la empresa.

\section{La experiencia bloqueada}

Este segundo tipo o figura de la experiencia laboral surge en los casos en que la promesa de integración inicial no se da por cumplida, sea por la fuerza de las vivencias cotidianas de malestar, abuso y sufrimiento, porque los costos subjetivos asociados a ser promovidos son mayores que los beneficios, o porque las expectativas de movilidad que generó inicialmente la empresa fueron superiores a lo ofrecido en realidad. En cualquier caso, la experiencia laboral en las tiendas se bloquea por la ausencia de perspectivas de realización en el trabajo y/o de proyectos laborales alternativos.

Aunque el trabajo en el retail ha significado el acceso a una cierta estabilidad y formalización en tanto empleados asalariados, en este tipo de experiencia laboral la socialización en las normas y valores empresariales nunca llegó a concretar una identificación con la empresa y la tienda. A su

11 Entrevista personal, 29 de marzo 2015, Santiago, Chile. 
vez, ni las condiciones asociadas al puesto de trabajo ni las inserciones laborales anteriores han permitido cristalizar una identidad de oficio asociada a la ocupación. Más bien, las categorías de identificación y pertenencia desde las cuales se dota de sentido y se orienta la acción en las tiendas se encuentran fuera del espacio de trabajo, principalmente en la familia. Así, la experiencia laboral se narra desde el lugar de un/a trabajador/a que lucha a diario en el mercado laboral por garantizar el sustento de su grupo familiar.

En gran medida por haber seguido trayectorias interrumpidas, con entradas y salidas del mercado laboral en función de ciclos de crianza y el cuidado del hogar, la relación que se establece con el trabajo acá es de carácter instrumental, concibiéndose sobre todo como un medio para sustentar a la familia y financiar la educación de los hijos, en quienes se deposita la promesa de una movilidad social futura que para ellos aparece bloqueada. Junto con el acceso a un empleo formal que garantiza un ingreso mensual regular, lo que más se valora del espacio laboral es la sociabilidad, las relaciones informales con los compañeros, jefes e incluso con los clientes.

Dichas relaciones -en forma similar a lo visto en estudios previos con trabajadores de supermercados (Stecher et al., 2010) - constituyen un importante soporte social y emocial en la vida de los trabajadores, el cual morigera y atenúa la vivencia de bloqueo y falta de perspectivas de desarrollo laboral. El trabajo en la tienda es, en consecuencia, tanto un medio de subsistencia como un lugar de encuentro y sociabilidad con otros.

En este tipo de experiencia, por sobre las dimensiones de agencia y autorrealización en el trabajo, predomina en la subjetivación el registro del padecimiento, la incertidumbre y el desgaste. Dada la gran importancia que posee la familia y particularmente los hijos para la construcción del sentido de la experiencia laboral, destaca el sufrimiento vinculado con las largas y atípicas jornadas que obligan a estar en el trabajo cuando se siente que se debería estar en el hogar, asumiéndose dicha ausencia, en especial en las trabajadoras mujeres y madres, como un gran costo y con grados importantes de culpabilidad. A ello se adiciona, en especial en el caso de las mujeres, que un porcentaje significativo de los tiempos libres se dedica al trabajo doméstico, lo cual refuerza la experiencia vital general de cansancio y desgaste ligado a extensas jornadas de trabajo productivo y reproductivo.

El bloqueo también se expresa en aquellas situaciones donde existiendo posibilidades de promoción, esta se rechaza. En efecto, aunque para algunos ser jefe, además de acceder a un cargo con mejor remuneración y estatus, es un reconocimiento al trabajo realizado y un desafío nuevo, para otros está asociado a la experiencia de trabajo-sin-fin que conlleva una jornada de 
entre 50 y 60 horas semanales, debido a que contractualmente se rigen por la normativa que se aplica a cargos sin supervisión directa, lo cual imposibilita disfrutar con mayor autonomía del propio tiempo y en especial de la familia. El rechazo, por tanto, es una manera de conservar estratégicamente la posición en la empresa, al constatar que los costos de ser jefe superan mucho los beneficios.

Asimismo, la experiencia de bloqueo también se observa en algunos trabajadores que se vieron atraídos por la oportunidad de hacer carrera en la empresa, tuvieron las posibilidades y decidieron ascender. En esos casos, la promesa de integración basada en la movilidad, que en un inicio pareció casi ilimitada, a medida que se avanzó se constató que se cerraba y/o se abría para otros que sí lograban seguir avanzando, pero que a juicio propio no tenían el suficiente mérito. La oferta de hacer carrera, por tanto, parece no ser igual a lo que se prometía, lo cual genera frustración y despierta una sensación de injusticia ante los anhelos que se vieron bloqueados, distanciándose el trabajador - una vez más, pero de otro modo- de la identificación con la empresa.

Esta figura de la experiencia social caracterizada por diferentes modalidades de bloqueo tiene lugar predominantemente entre los trabajadores adultos o de mayor edad, con cierta antigüedad en la organización, entre quienes tienen mayores responsabilidades familiares y con una expresión más fuerte en mujeres. A su vez, se observa cierta transversalidad en relación con los niveles ocupacionales, pudiendo ser experimentada por los cargos más bajos (cajera, asistente de venta, ordenador), como por vendedores o jefaturas intermedias, quienes vieron frustradas en algún momento sus expectativas de seguir ascendiendo, ser reconocidos y mejorar sus condiciones.

\section{La experiencia en tránsito}

Desde la experiencia que hemos denominado “en tránsito", la promesa de integración no resulta atractiva, y la socialización de la organización no alcanza a conquistar la subjetividad de los trabajadores. Aquello se debe principalmente porque compite con horizontes de sentido exteriores a la empresa, como la impronta de una cultura juvenil desde la cual el trabajo en la empresa es devaluado, y el imperativo extendido de obtener un título en la educación superior. Todo ello genera anhelos de realización que las empresas del rubro parecen no poder cumplir, produciéndose una forma de experiencia laboral caracterizada por el estar en tránsito hacia otro estado, lugar o posición. 
Como hemos señalado, el retail constituye una posibilidad de inserción en un empleo formal que es fácilmente accesible para los jóvenes, independientemente de su nivel educativo, experiencia laboral previa o su condición socioeconómica. No obstante, el empleo en el retail no es concebido por muchos de ellos como una inserción definitiva, sino como un lugar de tránsito en el cual no se trabajará para siempre. En efecto, a pesar de que el trabajo en el retail en muchos casos representa un avance en la trayectoria laboral de ciertos trabajadores jóvenes, sobre todo para aquellos con empleos esporádicos previos en el sector informal, con el paso del tiempo se va haciendo evidente que no es precisamente lo que quieren para sus vidas. Sin embargo, como ha sido de todas formas la puerta de entrada a una experiencia de integración al mercado laboral formal y a una organización moderna, aparece como el "piso" mínimo del cual no se puede descender y como una vía de acceso a otras posibilidades de desarrollo personal, de formación o de inserción laboral que antes no se vislumbraban.

Si la socialización de la empresa no logró concretarse del todo, fue porque no pudo competir con la influencia de otros referentes de sentido. Por un lado, una cultura juvenil a la cual no le seduce la idea de un trabajo que se presenta -en particular en los cargos de menor jerarquía y calificacióncomo rutinario, controlado y poco estimulante, que además obstruye el disfrute del tiempo libre con sus jornadas que terminan tarde y se trabaja los fines de semana. Por otro, el imperativo fuertemente asentado en la sociedad chilena de que los proyectos de vida y las posibilidades de ser "alguien en la vida” se cifran en el paso por la educación superior y la obtención de un título universitario.

La educación superior para el caso de los trabajadores de la empresa del retail aparece como "la" vía de desarrollo de un proyecto personal y de acceso a mejores condiciones de vida en Chile. Como señala una entrevistada: "En diciembre ya podría postular, acá, como vendedora o como cajera, tratar de bajar las horas de trabajo, ya, a veinticinco... iy poder estudiar!, porque no quiero estar toda la vida en retail" (Asistente, 22 años). ${ }^{12}$

De esta forma, en esta experiencia se combina de manera estratégica el esfuerzo por aprovechar y obtener los beneficios y posibilidades que ofrece en el presente el trabajo en las tiendas (aprendizajes, contactos, incentivos económicos), junto a una apuesta a futuro centrada en poder iniciar o terminar los estudios superiores y/o insertarse en un empleo de mayor estatus y remuneración. La vivencia de estar "en tránsito" lleva a que muchas veces este colectivo de trabajadores responda de forma errática a la lógica de los incentivos

12 Entrevista personal, 7 de diciembre 2014, Santiago, Chile. 
económicos que diseña la empresa, pues si bien tienen una orientación a obtenerlos, no están dispuestos a sobre-exigirse o a renunciar a ciertos proyectos personales por las exigencias de un trabajo en el cual no se proyectan. Por ello, incluso en quienes tienen buen desempeño y podrían optar a cierto ascenso dentro de la empresa, tienen plena claridad que su proyecto se juega en otros espacios (estudiar, buscar otro empleo con mejor horario y renta, etcétera).

El sentido de realización, en este contexto, se juega en la capacidad de desear, de proyectarse, en que se está construyendo un proyecto de vida y generando en forma autónoma recursos para poder llevarlo a cabo. Hay una distancia activa y un ejercicio crítico respecto a la industria y la tienda, centrados en experiencias de explotación o agobio laboral, pero sobre todo en la convición de que el trabajo en el retail es incompatible a largo plazo con un proyecto de certificación técnica o universitaria, concebido como el medio principal para lograr otras oportunidades laborales y una mejor posición social.

Sin embargo, con frecuencia la claridad y viabilidad de dicho proyecto no está del todo elaborada, existiendo muchas veces dudas en relación con qué estudiar, en qué tipo de institución, en qué momento y con cuáles medios. La indefinición parece comprometer la concreción efectiva del proyecto, generando que en ocasiones el tiempo en la empresa continúe avanzando y el trabajo vaya adquiriendo una ambigua consistencia entre provisorio y permanente.

La experiencia “en tránsito" se configura principalmente entre los trabajadores más jóvenes, con menos responsabilidades familiares que las de las otras figuras de experiencia, con mayor nivel educacional (liceos técnicos, educación técnica superior), con menor antigüedad en las tiendas y usualmente insertos en los puestos de trabajo con menor autonomía, mayor control directo y bajos reconocimientos simbólicos y materiales.

\section{Conclusiones}

El artículo propuso una reconstrucción del modo en que se despliegan las tres lógicas de la acción social descritas por Dubet (2007, 2010 y 2013) en el espacio de trabajo de las tiendas por departamento. Respecto a la lógica de la integración, se dio cuenta del modo en que para los trabajadores el ingreso a las tiendas configura una promesa de integración a un estatuto de empleo asalariado, a un espacio de sociabilidad, al desarrollo de destrezas, aprendizajes e incluso identidades de oficio, así como a ciertas posibilidades de promoción y movilidad. 
Respecto a la lógica de la estrategia, se dio cuenta del predominio, en general, de una lógica más bien defensiva y reactiva, orientada a mantener las posiciones alcanzadas y ajustar la conducta a los sistemas de incentivos que ofrecen los modelos de atención de las tiendas.

En cuanto a la lógica de la subjetivación, se dio cuenta de orientaciones a la acción basadas en sentimientos de autorrealización y desarrollo laboral en las tiendas, pero también vinculadas con un distanciamiento crítico y reflexivo respecto a la industria, así como a fuertes vivencias de sufrimiento y malestar en el trabajo, especialmente por las largas jornadas y el desgaste emocional y físico del trabajo de atención.

Posteriormente, se propusieron tres figuras de la experiencia social en el trabajo en las tiendas por departamento, que resultan de formas específicas de articulación de las lógicas de acción descritas. La experiencia comprometida da cuenta de un vínculo subjetivo al trabajo en que las promesas de integración desde la empresa aparecen significativamente logradas; en que la acción estratégica se orienta a mantener y aprovechar de forma virtuosa los beneficios e incentivos que los sistemas de gestión ofrecen; y en que predominan los componentes de autonomía, agencia y realización en la lógica de la subjetivación.

La experiencia bloqueada da cuenta de una modalidad de vínculo subjetivo al trabajo en el retail en la cual predomina una vivencia de no cumplimiento o cumplimiento parcial de las promesas y expectativas de integración que supone el ingreso al retail; en donde predominan orientaciones estratégicas basadas en la defensa de lo alcanzado y en un vínculo instrumental al empleo sin mayores proyecciones de carrera y con un foco en el sustento del grupo familiar; y en donde resaltan los aspectos de la subjetivación vinculados al padecimiento y el desgaste.

La experiencia en tránsito da cuenta de formas de integración y socialización laboral en la tienda parcialmente logradas, no tanto producto del bloqueo o el no cumplimiento de las expectativas, sino producto de la fuerza de otras orientaciones y referentes simbólicos. En ella los actores despliegan estrategias en pos de lograr aprovechar al máximo los beneficios que ofrece el empleo, pero con cierto distanciamiento subjetivo y teniendo como objetivo principal la salida de la tienda.

En la subjetivación predomina el distanciamiento crítico respecto a la tienda y a sí mismo, significando al trabajo en la empresa y al rol de empleado como momentos transitorios que tienen valor en tanto soportes para la concreción en el futuro de otros proyectos donde se juegan realmente los anhelos de realización personal. 
La descripción y análisis presentado constituye un doble aporte al campo de los Nuevos Estudios Laborales en América Latina. En primer lugar, contribuye al acumulado de estudios de caso empíricos sobre experiencias laborales en distintos sectores que han experimentado procesos de reorganización empresarial de corte flexibilizador en los últimos años. Lo hace, además, en una industria que tiene una alta importancia en la economía actual, dado su carácter intensivo en mano de obra, su enorme crecimiento y despliegue a partir de la consolidación de grandes holding empresariales, y su carácter paradigmático en términos de procesos de modernización empresarial del sector servicios.

En esta línea, los hallazgos presentados a partir del caso del retail en Chile constituyen un aporte al esfuerzo analítico de identificar y comprender ciertas formas prototípicas y transversales de la experiencia laboral de los individuos en el mundo del trabajo contemporáneo. La experiencia del compromiso a través de una fuerte integración a la organización y/o al oficio; la experiencia del bloqueo asociada a la descalificación, falta de posibilidades de desarrollo y construcción de sentido desde el rol de padre/madre jefa de hogar; y la experiencia en tránsito orientada por la expectativa de movilidad y la viviencia de la inserción laboral como algo pasajero, son tres formas de experiencia social y orientación subjetiva al trabajo que tienen similitudes con lo descrito en otros estudios empíricos de sociedades noratlánticas (Casey, 1995; Dubar, 2001; Strangleman y Roberts, 1999) y latinoamericanas (Soto, 2008; Stecher, 2012).

Estos estudios dibujan un paisaje del impacto de los procesos de modernización empresarial y flexibilización laboral en las grandes organizaciones del sector moderno y formal de la economía, dando cuenta de la fragmentación de las experiencias laborales y su segmentación en al menos tres grandes categorías o situaciones de trabajo:

El grupo (más bien minoritario) de aquellos trabajadores cuyo rol y competencias siguen siendo valiosos y reconocidos bajos los nuevos sistemas de gestión flexible, que se comprometen y ajustan a las expectativas y ofertas de sentido y desarrollo de carrera que hace la empresa, y que construyen experiencias e identidades a partir de contenidos directamente vinculados con su labor en el proceso de trabajo y su posición dentro de la organización.

El colectivo que en el marco de procesos de reorganización flexible, descualificación y precarización del empleo ve bloqueadas sus posibilidades de movilidad, desarrollo y reconocimiento, debiendo someterse a condiciones laborales juzgadas como adversas por la necesidad de subsistencia familiar y construyendo desde ahí un sentido del trabajo fuertemente instrumental. 
El grupo que se inserta a la organización -por lo general jóvenes y bajo la figura de proyectos o empleo part time - con un horizonte temporal acotado, sin interés en desarrollar un oficio o hacer carrera en la empresa, con la aspiración de seguir estudiando y moverse en el mercado laboral y que se ajusta desde esa posición subjetiva a los sistemas de gestión flexible que le ofrece la organización.

Estas distintas formas de experiencia social en el trabajo son, por supuesto, móviles - pudiendo un individuo transitar entre ellas en función de cambios en la organización o de acontecimientos biográficos- se articulan de formas diferenciadas en distintos sectores productivos y declinan en modos singulares según los niveles de calificación de la fuerza de trabajo y sus anclajes estructurales (género, generación, etnia) en el orden social.

En segundo lugar, el artículo contribuye, a partir de la presentación y aplicación de la perspectiva teórica de la sociología de la experiencia de Dubet (2007, 2010 y 2013), a enriquecer el arsenal conceptual de los Estudios del Trabajo en América Latina respecto al estudio de la dimensión subjetiva de las transformaciones sociolaborales. El modelo de la experiencia social y las lógicas de acción permiten un análisis situado, procesual y complejo de la vida social y del mundo del trabajo, en el cual se reconocen tanto el peso de las dimensiones sistémicas y estructurales, como el rol central de los actores en la producción del sentido de sus experiencias laborales y en la orientación de su acción a través de la articulación reflexiva de lógicas de acción diferentes.

Como lo ilustran las tres figuras o tipos de experiencia descritas para las tiendas de departamentos, analizar las experiencias sociales y la subjetividad de los actores laborales en el mundo del trabajo exige reconstruir tanto las condiciones y características "objetivas" de una industria o escenario laboral, como el modo en que los individuos al interior de dichas condiciones tecno-socio-productivas -y a la luz de sus trayectos biográficos y sus anclajes múltiples en la estructura social (género, generación, clase, etnia) - articulan permanentemente lógicas de acción heterogénas para enfrentar desafíos cotidianos, vincularse con otros y elaborar sentidos de sí mismos como trabajadores.

De este modo, la utilización de la Sociología de la Experiencia de Dubet (2007, 2010 y 2013) como marco para la investigación empírica puede contribuir a profundizar nuestra comprensión de los escenarios laborales y sus transformaciones contemporáneas en América Latina, a partir del análisis de las diversas formas de vínculo subjetivo y experiencia social que construyen los actores en los heterogéneos mundos del trabajo de la región. 
Queda como desafío teórico-conceptual seguir explorando y discutiendo las articulaciones (diferencias y similitudes) entre la perspectiva de la sociología de la experiencia de Dubet y otros marcos conceptuales -identidades y culturas laborales, configuraciones subjetivas, formas de reconocimiento, acción colectiva, gubernamentalidad y subjetivación, etc.- actualmente utilizados en América Latina para el análisis de las dimensiones subjetivas de las transformaciones económicas y sociolaborales.

Resulta fundamental, por último, seguir avanzando en formas de conceptualización de las experiencias laborales que logren articular mejor sus dimensiones individuales y colectivas, que visibilicen sus implicancias sociopolíticas en el marco de la relaciones capital-trabajo y que iluminen la centralidad de la dimensión subjetiva para comprender el mundo del trabajo y sus transformaciones contemporáneas.

\section{Referencias}

Antunes, Ricardo (2003), ¿Adiós al trabajo?, Brasil: Cortez Editora.

Araujo, Kathya y Martuccelli, Danilo (2012), Desafios comunes. Retratos de la sociedad chilena y sus individuos, Chile: LOM.

Battistini, Osvaldo (2004), El trabajo frente al espejo, Argentina: Prometeo.

Boltanski, Luc y Chiapello, Eve (1998), El nuevo espiritu del capitalismo, España: Akal.

Calderón, Álvaro (2006), "El modelo de expansión de las grandes cadenas minoristas en Chile", en Revista CEPAL, núm. 90, Chile: Comisión de Económica para Latinoamérica y el Caribe.

Castells, Manuel, (2001), La era de la información, España: Alianza.

Casey, Catherine (1995), Work, self and society: after industrialism, Estados Unidos: Routledge.

De la Garza, Enrique (2000), Tratado latinoamericano de sociología del trabajo, México: Fondo de Cultura Económica.

Domingues, José (2009), La modernidad contemporánea en América Latina, Argentina: Siglo XXI.

Du Gay, Paul (1996), Consumption and identity at work, Inglaterra: Sage.

Dubar, Claude (2001), "El trabajo y las identidades profesionales y personales", en Revista Latinoamericana de Estudios del Trabajo, núm. 13, Argentina: Asociación Latinoamericana de Estudios del Trabajo.

Dubet, François (2006), Injustices: L'expérience des inégalités au travail, Francia: Le Seuil.

Dubet, François (2007), La experiencia sociológica, España: Gedisa.

Dubet, François (2010), Sociología de la experiencia, España: Centro de Investigaciones Sociológicas.

Dubet, François (2013), El trabajo de las sociedades, España: Amorrortu.

Flick, Uwe (2004), Introducción a la investigación cualitativa, España: Morata.

Fundación Sol (2016), Industrias de elaboración de alimentos y bebidas, retail y sector financiero, Chile: Fundación SOL. 
Convergencia Revista de Ciencias Sociales, vol. 27, 2020, Universidad Autónoma del Estado de México

Gálvez, Telma et al. (2009), "Holdings y tiendas: La trama que multiplica las ventas", en Cuaderno de Investigación, núm. 36, Chile: Dirección del Trabajo, Gobierno de Chile. Martuccelli, Danilo (2007), Cambio de Rumbo: la Sociedad a Escala del Individuo, Chile: LOM.

Martuccelli, Danilo y De Singly, François (2012), Las sociologías del individuo, Chile: LOM.

Ramos, Claudio (2009), La trasformación de la empresa chilena. Una modernización desbalanceada, Chile: Universidad Alberto Hurtado.

Soto, Álvaro [ed.] (2008), Flexibilidad laboral y subjetividades. Chile: LOM y Universidad Alberto Hurtado.

Soto, Álvaro (2015), “Escenarios del trabajo, una aproximación a la heterogeneidad del trabajo contemporáneo en Latinoamérica”, en Revista de Estudios Sociales, núm. 51, Colombia: Universidad de los Andes. DOI: https://dx.doi.org/10.7440/ res51.2015.15 Disponible en: https://revistas.uniandes.edu.co/doi/full/10.7440/ res51.2015.15, [05 de mayo de 2018].

Stecher, Antonio y Martinic, Rodolfo (2018), "La descualificación del trabajo en tiendas por departamento", en Psicoperspectivas, núm. 3, Chile: Pontificia Universidad Católica de Valparaíso.

Stecher, Antonio (2012), "Perfiles Identitarios de Trabajadores de Grandes Empresas del Retail en Santiago de Chile: Aportes psicosociales a la comprensión de las identidades laborales", en Revista PSYKHE, vol. 21, núm. 2, Chile: Pontificia Universidad Católica de Chile.

Stecher, Antonio (2013), "Un modelo crítico-interpretativo para el estudio de las identidades laborales. Contribuciones a la investigación psicosocial sobre trabajo y subjetividad en América Latina”, en Universitas Psychologica, núm. 124, Colombia: Universidad Javeriana. DOI http://dx.doi.org/10.11144/Javeriana.UPSY12-4.mcie Disponible en: https://revistas.javeriana.edu.co/index.php/revPsycho/article/view/6505 [15 de abril de 2018].

Stecher, Antonio et al. (2010), "Condiciones y experiencias de trabajo en la sala de venta de un supermercado", en Polis, vol. 9, núm. 27, Chile. DOI: http://doi.org/10.4067/ S0718-65682010000300024 Disponible en: https://scielo.conicyt.cl/scielo. php?script $=$ sci_arttext $\&$ pid $=$ S0718-65682010000300024\&lng=en\&nrm $=$ iso $\&$ tlng $=$ en $[15$ de abril de 2018].

Strangleman, Tim y Roberts, Ian (1999), "Looking through the Window of Opportunity: The Cultural Cleansing of Workplace Identity”, en Sociology, vol. 33, núm. 1 DOI: https://doi.org/10.1177/S0038038599000036 Disponible en: https://journals. sagepub.com/doi/10.1177/S0038038599000036 [24 de marzo de 2018].

Strauss, Anselm y Corbin, Juliet (2002), Bases de la investigación cualitativa: Técnicas y procedimientos para desarrollar la teoría fundamentada, Colombia: Universidad de Antioquía.

Todaro, Rosalba y Yáñez, Sonia (2004), El trabajo se transforma: relaciones de producción y relaciones de género, Chile: Centro de Estudios de la Mujer.

Wrigley, Neil y Lowe, Michelle (2010), The Globalization of Trade in Retail Services. Report Commissioned by the OECD, Francia: Organización para la Cooperación y el Desarrollo Económicos. 
Rodolfo Martinic. Doctorante en Sociología, École des Hautes Études en Sciences Sociales (EHESS), Francia. Magíster en Psicología social, Universidad Diego Portales, Chile. Sociólogo, Pontificia Universidad Católica de Chile. Línea de investigación principal: el estudio del trabajo en el mundo contemporáneo, a través de la consideración de la escala individual, organizacional e institucional del fenómeno. Publicaciones recientes: Martinic, Rodolfo (2019), “¿Qué hay tras la permanencia universitaria? Los cambios más significativos en el acompañamiento académico”, en Educação e Pesquisa, núm. 45, DOI: https://dx.doi.org/10.1590/s1678-4634201945190724; Stecher, Antonio y Martinic, Rodolfo (2018), "La descualificación del trabajo en tiendas por departamento", en Psicoperspectivas, núm. 3, Chile: Pontificia Universidad Católica de Valparaíso; Martinic, Rodolfo y Bravo, Valentina (2011), "Sostenidos en colectivo. El proceso de individuación de trabajadores en campamentos”, en Revista CIS, vol. 9, núm. 15, Chile: Fundación Techo.

Antonio Stecher. Doctor en Psicología Social por la Universidad Autónoma de Barcelona. Psicólogo y Magíster en Filosofía Política por la Universidad de Chile. Profesor Facultad de Psicología, Universidad Diego Portales, Chile. Director alterno e investigador asociado del Centro Núcleo Milenio Autoridad y Asimetrías de Poder/Millennium Nucleus Center Authority and Power Asymmetries. Línea principal de investigación: las transformaciones del trabajo y las subjetividades laborales en la modernidad contemporánea en América Latina. Publicaciones recientes: Bank, Carolina Kenny, Briget y Stecher, Antonio (2018), Wal-Mart in the Global South: Workplace Culture, Labor Politics, and Supply Chains, Estados Unidos: University of Texas Press; De la Fabián, Rodrigo \& Stecher, Antonio (2017), "Positive psychology’s promise of happiness: a new form of human capital in contemporary neoliberal governmentality", en Theory \& Psychology, vol. 27, núm. 5, Estado Unidos: Sage; Stecher, Antonio (2015), "La empresa flexible como dispositivo de gobierno. Aportes de la Analítica de la Gubernamentalidad al estudio de las subjetividades laborales en América Latina”, en Universitas Psychologica, vol. 14, núm. 5, Colombia: Universidad Javeriana. 